Reprod. Nutr. Dévelop., 1984, 24 (4), 405-418.

\title{
Localisation immunocytochimique et analyse quantitative des cellules gonadotropes et thyréotropes de l'adénohypophyse de la Cane de Barbarie (Cairina moschata L.) au cours du cycle annuel ou après ovariectomie
}

\author{
P. JANOD, C. R. MARCHAND, Claude COLARD。
}

Laboratoire de Zoologie et Embryologie, L.A. CNRS 071049, Faculté des Sciences, place Maréchal-Leclerc, 25030 Besançon Cedex, France.

Summary. Immunocytochemical localization and quantitative analysis of $\mathrm{LH}$, FSH and TSH cells in the adenohypophysis of the female Muscovy duck (Cairina moschata L.).

Two cell types have been immunocytochemically identified in the adenohypophysis of Muscovy duck females, intact or with the left ovary removed, and killed at different times of the annual cycle. Anti-chicken LH, anti-chicken FSH, anti-beta bovine TSH antisera and the peroxidase anti-peroxidase (PAP) method were used.

The gonadotrophes (LH and FSH cells) were scattered in the pars distalis whereas the thyreotrophes (TSH cells) were exclusively located in the cephalic lobe of the pituitary gland.

All the cells were more or less stimulated by early left ovariectomy. Quantitative analysis (diameter, surface and number of immunoreactive cells) led to a comparison of the variations of each cell type at different times of the cycle in the controls as well as in operated ducks.

\section{Introduction.}

Les cellules de l'adénohypophyse des Oiseaux ont d'abord été étudiées par les techniques de colorations topographiques et histochimiques que sont le tétrachrome de Herlant (Herlant, 1960 ; Herlant et al., 1960) et le bleu alcian APS (Tixier-Vidal et Benoit, 1962; Marchand et al., 1969 ; Marchand, 1972 ; revue dans Tixier-Vidal et Follett, 1973) chez le Canard mâle. D'autre part, des études ultrastructurales réalisées par Mikami et al. (1975), Mikami (1980), Tixier-Vidal (1972), Tixier-Vidal et al. (1972), revue dans Marchand (1978), ont fourni des arguments indirects pour identifier un type cellulaire au plan morphofonctionnel.

La mise au point des techniques immunocytochimiques a permis récemment de mieux connaître la cytophysiologie adénohypophysaire des Oiseaux (Hansen et Hansen, 1977 ; Marchand, 1978 ; Marchand et al., 1975, 1976, 1978 ; Marchand 
et Sharp, 1977) en utilisant des anticorps dirigés contre les molécules hormonales recherchées ; elles ont fourni des éléments directs d'identification des différents types cellulaires de la glande pituitaire.

Dans le cadre d'une étude des causes et des conséquences de la stérilité des Canes hybrides stériles issues du croisement Canard Pékin mâle $\times$ Cane de Barbarie (Gomot et Ardiet, 1966a, b ; Gomot et al., 1966 ; Marchand, 1972 ; Deray, 1978), nous nous sommes proposés d'établir la typologie des cellules adénohypophysaires des espèces parentes, et en premier lieu de la Cane de Barbarie, jamais étudiée à ce jour.

Les animaux ont été observés dans différents états physiologiques naturels (cycle annuel en fonction de la photopériode) ou expérimentaux (ovariectomie gauche). L'existence chez les Oiseaux d'une photosensibilité très marquée au cours du cycle saisonnier (Benoît, 1934 ; Tixier-Vidal et Assenmacher, 1961a, b ; Tixier-Vidal et al., 1962, 1968) se répercutant par l'intermédiaire de l'hypophyse sur le tractus génital, nous a amenés à étudier les différents aspects des cellules adénohypophysaires au cours du cycle annuel.

L'effet de l'ovariectomie sur la cytophysiologie adénohypophysaire est un moyen d'investigation indispensable pour repérer les cellules gonadotropes et pour visualiser leur comportement après suppression du " feed back ".

Nous avons réalisé ce travail en utilisant les anticorps anti-LH totale de Poulet, anti-FSH de Poulet et anti-bêta TSH bovine. Nous avons en même temps caractérisé et analysé les cellules thyréotropes dans différentes conditions physiologiques afin de savoir si elles répondent à l'ovariectomie.

\section{Matériel et méthodes.}

1) Les animaux. - De jeunes Canes de Barbarie à l'éclosion, obtenues auprès d'un élevage sélectionné, sont élevées dans une animalerie climatisée, puis, à l'état adulte, elles sont placées dans des enclos extérieurs où elles sont soumises aux conditions climatiques de la région. L'étude porte sur des lots de 4 animaux.

2) Les méthodes. - L'ovariectomie gauche est réalisée 15 à 20 jours après éclosion par le Prof. Gomot. Les animaux sont sacrifiés 1 à 2 ans après l'opération. L'hypophyse des Canes sacrifiées par décapitation est rapidement prélevée et fixée au Bouin Hollande sublimé durant une semaine. Après inclusion dans le Paraplast, des coupes sériées sagittales de 5 microns sont préparées. A la suite du déparaffinage et du lavage à l'eau courante, les coupes sont rincées dans la solution tampon phosphate (PBS) 0,01 M, NaCl 0,15 M, pH 7,2.

L'immunocytochimie est réalisée selon la technique qui utilise les complexes solubles de PAP (Sternberger et al., 1970). L'anti-LH totale fraction CM2 obtenu chez le Lapin a été fournie par le Docteur P. J. Sharp (Agricultural Research Council's Poultry Research Centre, Edinburgh, Scotland) qui en a vérifié la spécificité (Sharp, 1974). L'anti-FSH de Poulet obtenue chez le Lapin a été gracieusement fournie par le Docteur S. Ishii (Department of Biology, School of Education, 
Waseda University, Shinjuku ku, Tokyo, Japan) qui en a vérifié la spécificité (Sakai et Ishii, 1980). L'anti-bêta TSH bovine a été contrôlée et fournie par le Docteur Pierce (Department of Biological Chemistry, Los Angeles, CA 90024, USA).

Les dilutions des immunsérums dans le tampon PBS ont été de : $1 / 2000$ ou $1 / 4000$ pour l'anti-H; $1 / 1000$ ou $1 / 2000$ pour l'anti-FSH et $1 / 500$ pour l'antiTSH. La durée de ce contact est d'une nuit à $4{ }^{\circ} \mathrm{C}$.

Le second immunsérum est un anti-gamma globulines de Lapin obtenu chez la Chèvre ; il est dilué au 1/60. La peroxydase anti-peroxydase obtenue chez le Lapin est diluée au $1 / 200$; la présence de la peroxydase est révélée par la diaminobenzidine (DAB $5 \mathrm{mg}$ dans $10 \mathrm{ml}$ PBS + 0,01\% $\mathrm{H}_{2} \mathrm{O}_{2}$ ).

L'analyse quantitative a été réalisée à l'aide d'un analyseur d'images Kontron MOP 3 sur photographies de grandissement 375 ou 600 . Les paramètres cellulaires sont mesurés en $\mu \mathrm{m}^{2}$ (surface projetée) ou en $\mu \mathrm{m}$ (diamètre maximal projeté). L'étude est réalisée selon le schéma d'une distribution de population ; les résultats sont comparés par le test de Student (Test T, Schwartz, 1963) au calculateur Hewlett Packard.

\section{Résultats.}

Les marquages immunocytochimiques sur coupes contiguës avec les différents immunsérums utilisés montrent (fig. 1, 2, 3, PI. I) que :

- L'anti-bêta TSH bovine (fig. 3, PI. I) marque exclusivement les cellules produisant la TSH qui se localisent uniquement dans le lobe céphalique de la pars distalis.

- L'anti-LH totale de Poulet (fig. 1, PI. I) sur coupe adjacente met en évidence une autre population cellulaire distincte des cellules TSH : ce sont les cellules gonadotropes réparties dans les deux lobes de la glande pituitaire. L'immunsérum marque également les cellules révélées par l'anti-TSH et celles marquées par I'anti-FSH; notons toutefois qu'une population cellulaire ne fixe que l'antiLH.

- L'anti-FSH de Poulet (fig. 2, PI. 1) marque les cellules gonadotropes déjà révélées par I'anti-LH, mais aussi une population spécifiquement FSH positive.

L'immunocytochimie met donc en évidence deux populations cellulaires dans l'adénohypophyse de la Cane de Barbarie :

\section{PLANCHE I}

Coupes contiguës d'adénohypophyse de Cane de Barbarie traitées en immunocytochimie par la technique à la PAP (Sternberger) avec des immunsérums Fig. 1 : anti-LH totale de Poulet, Fig. 2 : antiFSH de Poulet, Fig. 3 : anti- $\beta$ TSH bovine.

La technique met en évidence des cellules marquées par deux immunsérums ; certaines cellules ne sont immunoréactives que vis-à-vis d'un immunsérum.

(Toutes les photos sont au grossissement $\times 600$ ). 

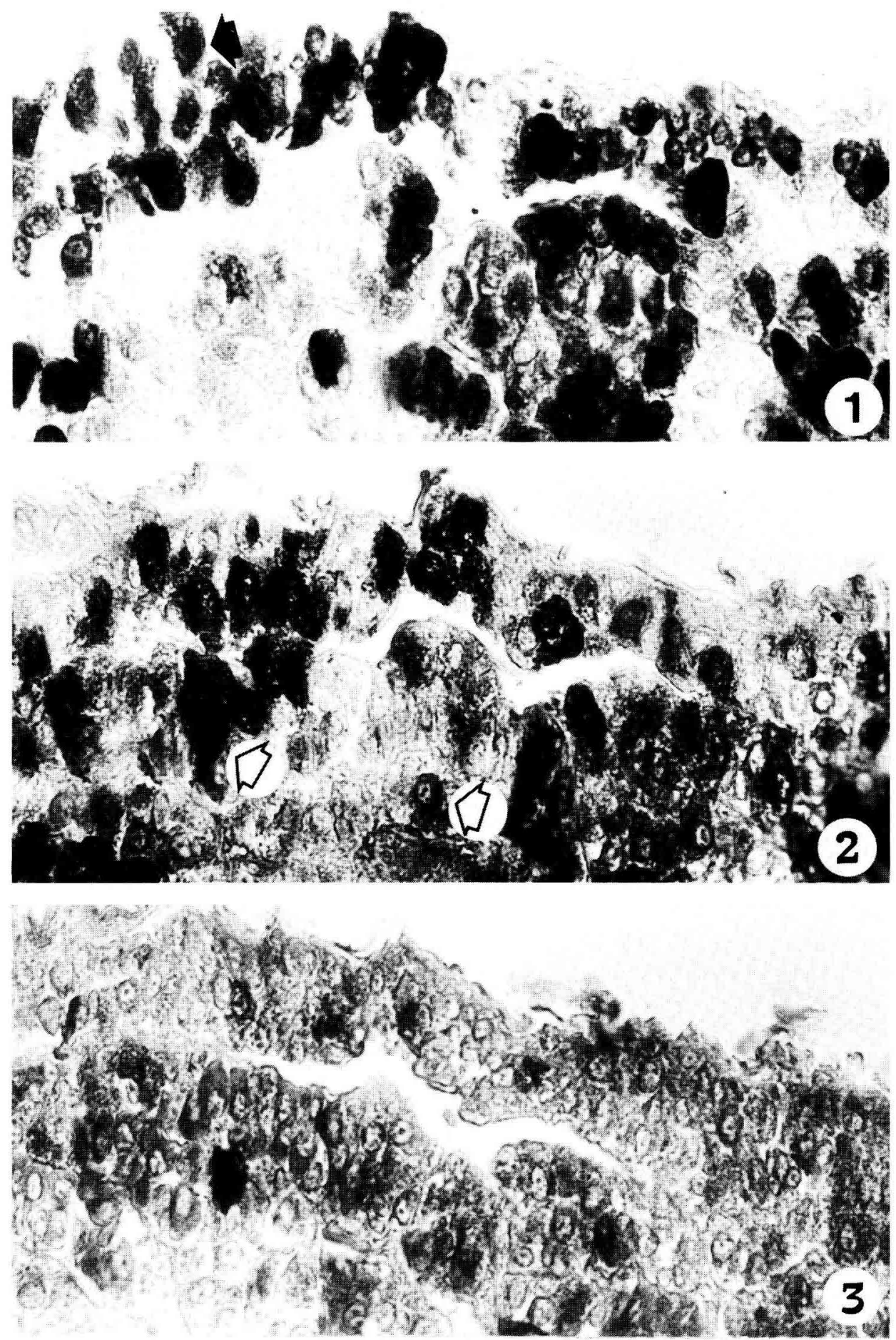


\section{1) Les cellules gonadotropes}

a) Celles qui sont marquées par l'anti-LH, réparties dans les deux lobes de la pars distalis : les cellules LH positives de la Cane de Barbarie témoin sacrifiée en photopériode longue (5 876 cellules $/ \mathrm{mm}^{2}$; tabl. 1). Ces cellules se répartissent en grande partie dans le lobe caudal à proximité du lobe nerveux et dans la zone ventrale du lobe céphalique. Elles sont de section circulaire et possèdent une disposition palissadique au sein des acini (fig. 1, PI. II ; fig. 1, PI. III). L'étude statistique de cette population (fig. 1) montre que les paramètres taille et surface cellulaire projetées sont réduits de $30 \%$ chez l'animal examiné en photopériode longue.

TABLEAU 1

Analyse quantitative des cellules $\mathrm{LH}^{+}, \mathrm{FSH}^{+}$et $\mathrm{TSH}^{+}$de la Cane de Barbarie (Cairina moschata L.).

\begin{tabular}{|c|c|c|c|c|c|c|c|c|c|}
\hline & \multicolumn{3}{|c|}{ LH } & \multicolumn{3}{|c|}{$\mathrm{FSH}$} & \multicolumn{3}{|c|}{ TSH } \\
\hline Animal & $\begin{array}{c}\text { Nombre } \\
/ \mathrm{mm}^{2}\end{array}$ & $\underset{\mu \mathrm{m}}{\mathrm{D} \max }$ & $\mathrm{S} \mu \mathrm{m}^{2}$ & $\begin{array}{c}\text { Nombre } \\
/ \mathrm{mm}^{2}\end{array}$ & $\underset{\mu \mathrm{m}}{\mathrm{D} \max }$ & $\mathrm{S} \mu \mathrm{m}^{2}$ & $\begin{array}{c}\text { Nombre } \\
/ \mathrm{mm}^{2}\end{array}$ & $\underset{\mu \mathrm{m}}{\mathrm{D} \max }$ & $\mathrm{S} \mu \mathrm{m}^{2}$ \\
\hline $\begin{array}{c}\text { Témoin } \\
\text { sacrifié en } \\
\text { photopériode } \\
\text { courte }\end{array}$ & 3339 & $\begin{array}{c}8,77 \\
\pm \quad 1,37\end{array}$ & $\begin{array}{c}50,07 \\
\pm \quad 11,2\end{array}$ & 3725 & $\begin{array}{c}8,73 \\
\pm \quad 1,63\end{array}$ & $\begin{array}{c}44,07 \\
\pm 12,1\end{array}$ & 64 & $\begin{array}{l}8,64 \\
2,13\end{array}$ & $\begin{array}{c}41,02 \\
\pm \quad 13,6\end{array}$ \\
\hline $\begin{array}{c}\text { Témoin } \\
\text { sacrifié en } \\
\text { photopériode } \\
\text { longue }\end{array}$ & 5876 & $\begin{array}{r}6,90 \\
\pm 0,99\end{array}$ & $\begin{array}{c}30,42 \\
\pm \quad 8,8\end{array}$ & 3379 & $\begin{array}{c}10,51 \\
\pm \quad 1,95\end{array}$ & $\begin{array}{c}63,56 \\
\pm 14,7\end{array}$ & 178 & $\begin{array}{r}10,49 \\
\pm \quad 1,6\end{array}$ & $\begin{array}{r}62,25 \\
\pm \quad 15,5\end{array}$ \\
\hline $\begin{array}{l}\text { Ovariectomisé } \\
\text { sacrifié en } \\
\text { photopériode } \\
\text { courte }\end{array}$ & 5022 & $\begin{array}{c}11,19 \\
\pm 2,04\end{array}$ & $\begin{array}{c}68,78 \\
\pm \quad 13,1\end{array}$ & 5405 & $\begin{array}{c}10,72 \\
\pm \quad 1,71\end{array}$ & $\begin{array}{c}64,80 \\
\pm \quad 15,4\end{array}$ & 258 & $\begin{array}{r}10,58 \\
\pm \quad 2,2\end{array}$ & $\begin{array}{l}53,49 \\
\pm \quad 13,3\end{array}$ \\
\hline $\begin{array}{c}\text { Ovariectomisé } \\
\text { sacrifié en } \\
\text { photopériode } \\
\text { longue }\end{array}$ & 5312 & $\begin{array}{l}12,09 \\
\pm 1\end{array}$ & $\begin{array}{c}75,83 \\
\pm \quad 27,8\end{array}$ & 6429 & $\begin{array}{l}11,67 \\
\pm 2,44\end{array}$ & $\begin{array}{l}79,94 \\
\pm 20,1\end{array}$ & 233 & $\begin{array}{r}10,24 \\
\pm 2,2\end{array}$ & $\begin{array}{c}65,71 \\
\pm \quad 18,0\end{array}$ \\
\hline
\end{tabular}

\section{PLANCHE II}

Coupes d'adénohypophyses traitées en immunocytochimie par la technique à la PAP (Sternberger) avec des immunsérums anti-LH totale de Poulet (Fig. 1,2), anti-FSH de Poulet (Fig. 3, 4) et anti$\beta$ TSH bovine (Fig. 5, 6), prélevées chez des Canes de Barbarie témoin (Fig. 1, 3, 5) ou ovariectomisées (Fig. 2, 4,6) et sacrifiées en photopériode longue.

Chez les Canes ovariectomisées, on remarque l'hypertrophie des cellules marquées par les trois immunsérums, ainsi que l'augmentation du nombre des cellules immunoréactives (Fig. 2, 4, 6). (Toutes les photos sont au grossissement $\times 600$ ). 

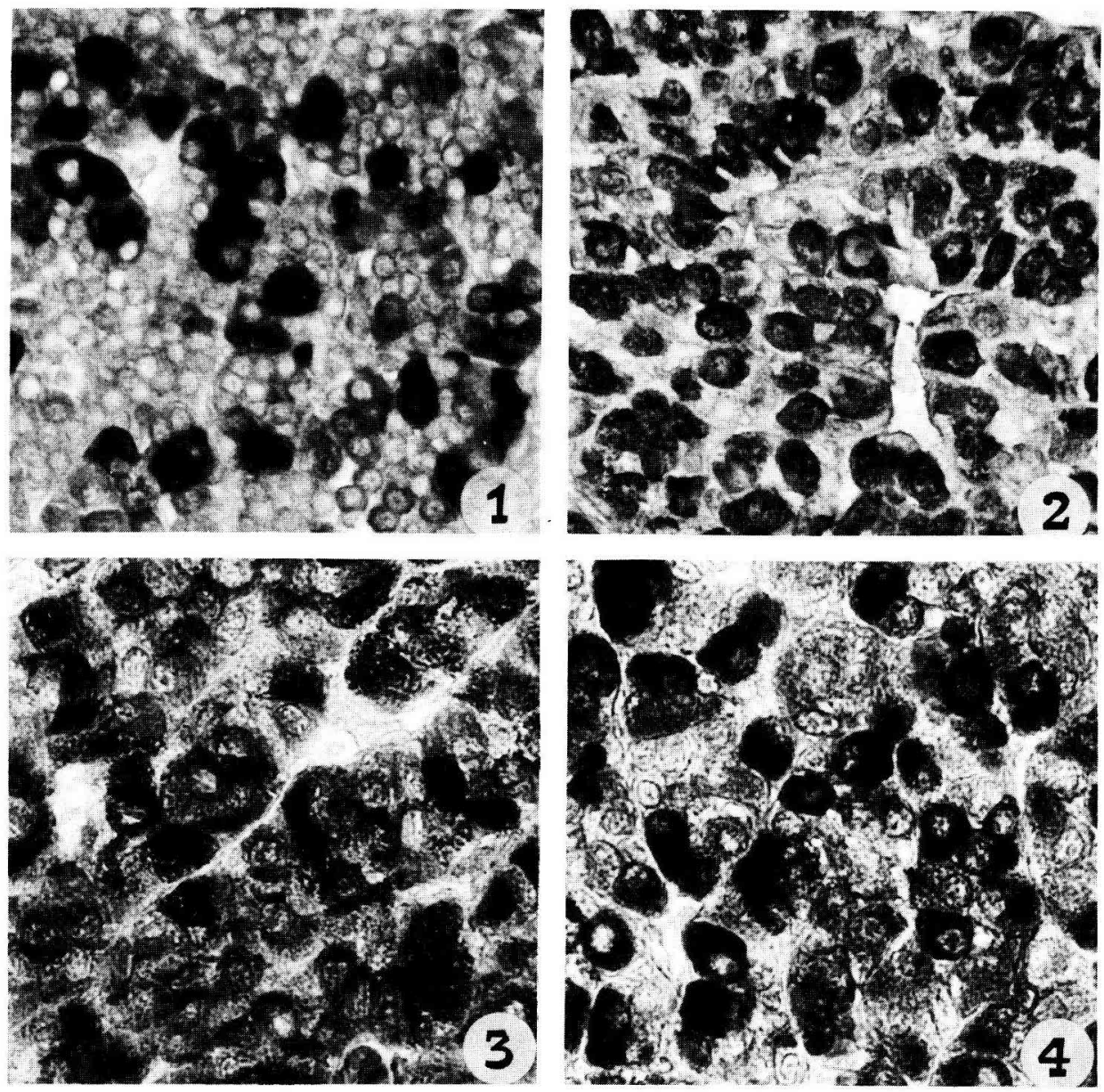

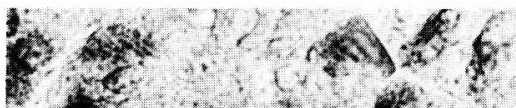
3.t.

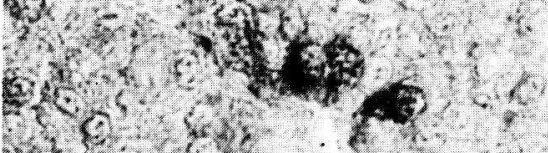

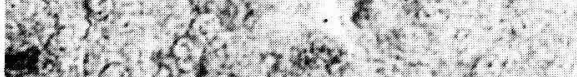

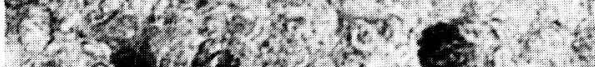

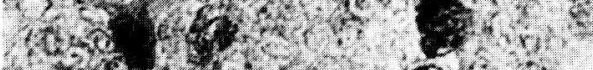

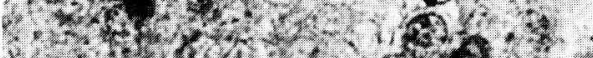

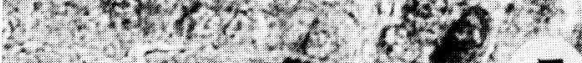

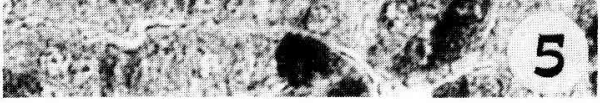

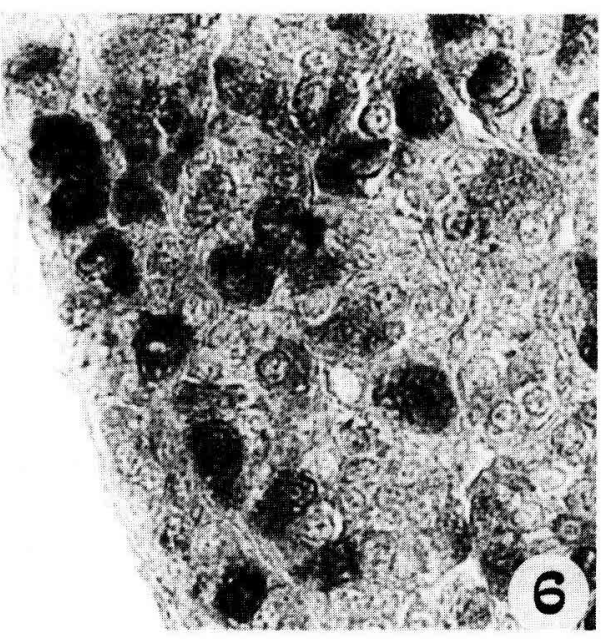




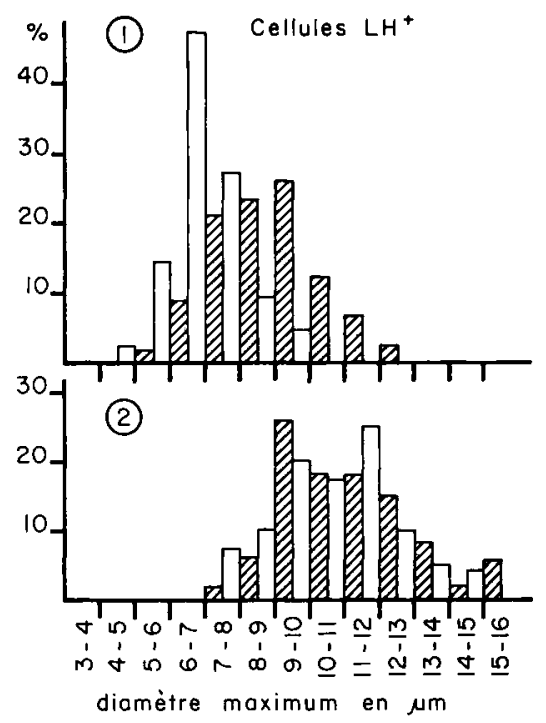

FIG. 1 et 2. - Histogrammes de la distribution des cellules LH chez une Cane de Barbarie témoin

(Fig. 1) et ovariectomisée (Fig. 2). Photopériode courte $[/ \triangle$. Photopériode longue

L'ovariectomie gauche de la Cane de Barbarie provoque une hypertrophie des cellules gonadotropes entraînant l'hyperplasie de la glande (fig. 2, PI. II ; fig. 2. PI. III). Le nombre de cellules LH positives chez l'animal ovariectomisé (5022 cellules $/ \mathrm{mm}^{2}$ ) représente une augmentation de $50 \%$ par rapport au témoin (tabl. 1). L'étude quantitative (fig. 2) met en évidence une augmentation de $40 \%$ de la taille et de la surface cellulaire projetées chez la Cane ovariectomisée sacrifiée en photopériode courte par rapport au témoin. Chez l'animal sacrifié en photopériode longue (fig. 2), le nombre de cellules n'augmente pas, la taille et la surface cellulaire projetées doublent par rapport à l'animal témoin.

b) L'anti-FSH de Poulet marque les cellules gonadotropes qui se localisent dans le lobe caudal et dans le lobe céphalique de l'adénohypophyse de la Cane de Barbarie. Le nombre de cellules FSH immunoréactives de la Cane témoin sacrifiée en photopériode longue ( 3379 cellules $/ \mathrm{mm}^{2}$ ) n'augmente pas par rapport à l'animal témoin sacrifié en photopériode courte $\left(3725\right.$ cellules $\left./ \mathrm{mm}^{2}\right)$. L'étude quan-

\section{PLANCHE III}

Coupes d'adénohypophyses traitées en immunocytochimie par la technique à la PAP (Sternberger) avec des anti- $\mathrm{LH}$ totale de Poulet (Fig. 1 et 2), anti-FSH de Poulet (Fig. 3 et 4) et anti- $\beta$ TSH bovine (Fig. 5 et 6), prélevées chez des Canes de Barbarie témoin (Fig. 1, 3, 5) ou ovariectomisées (Fig. 2, 4, 6) et sacrifiées en photopériode courte.

Chez les Canes ovariectomisées, on note l'hyperactivation des cellules marquées par les immunsérums anti-LH, anti-FSH et anti-TSH (Fig. 2, 4,6).

(Toutes les photos sont au grossissement $\times 600$ ). 

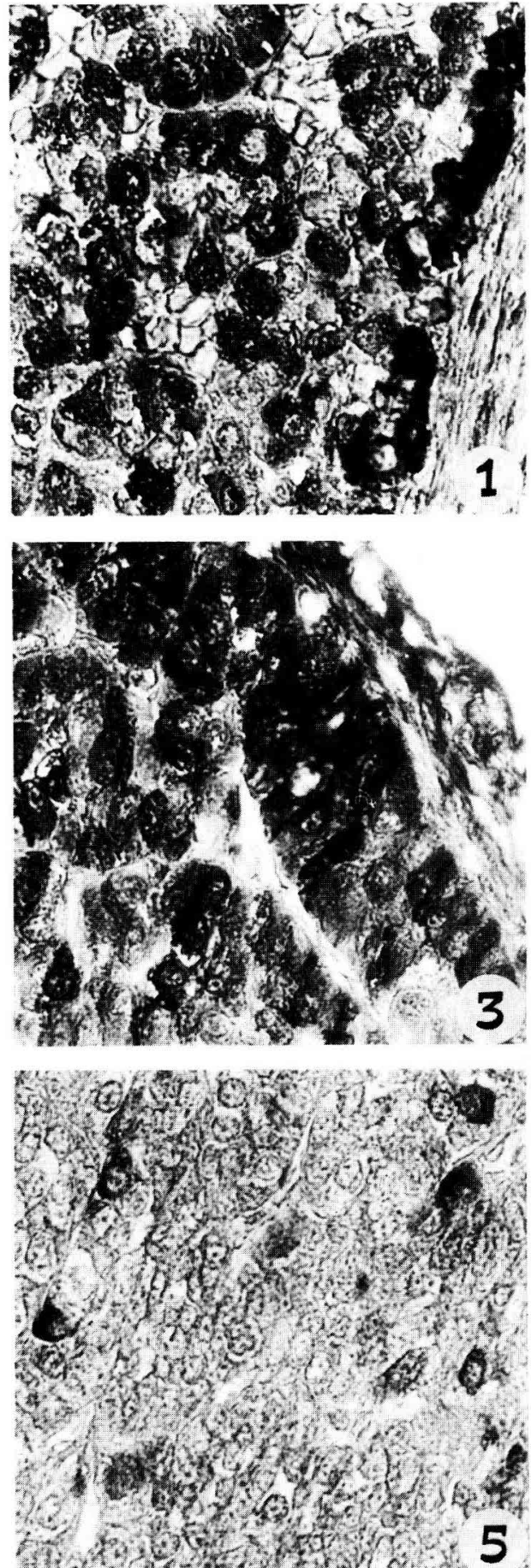

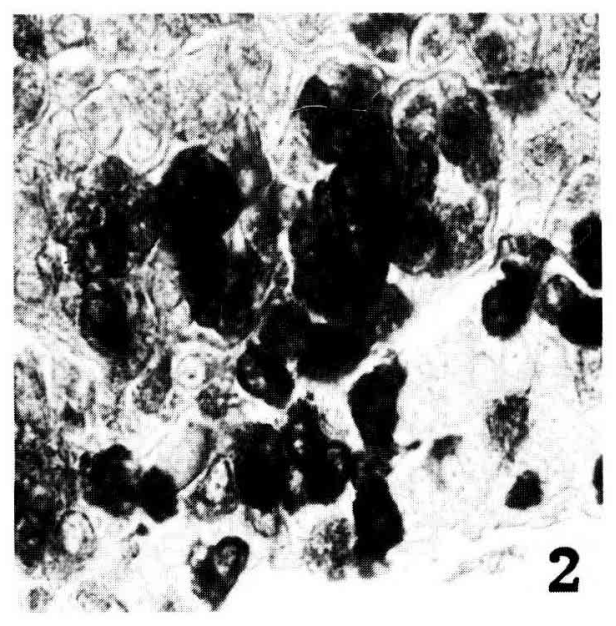

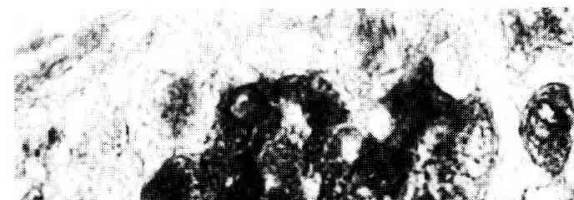

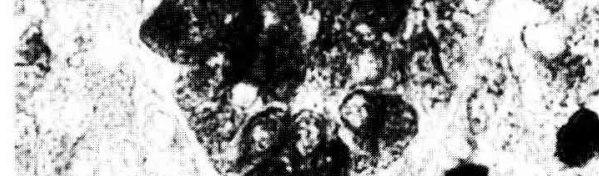

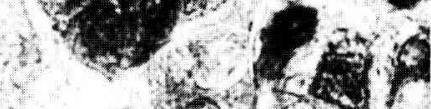

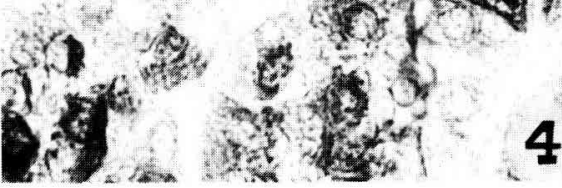

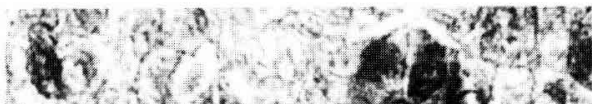

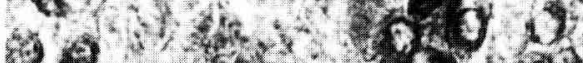

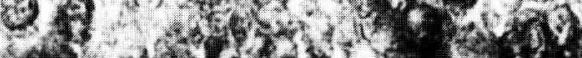

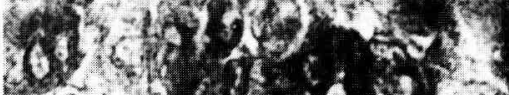

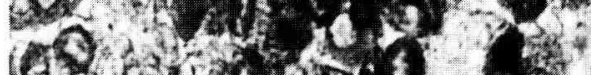

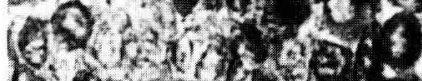

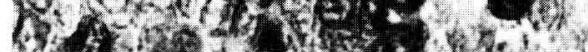
cal -3$)$ w.

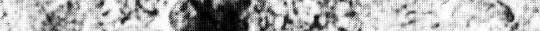

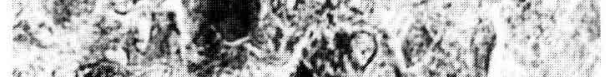

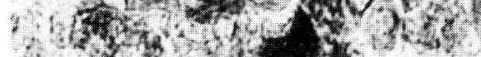

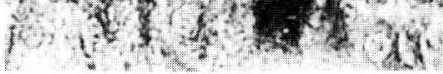


titative (fig. 3) montre que la taille et la surface cellulaire projetées augmentent durant la photopériode longue chez la Cane témoin.

L'ovariectomie gauche de la Cane de Barbarie entraîne une hypertrophie des cellules FSH immunoréactives, ainsi que l'hyperplasie de la glande pituitaire (fig. 4, PI. II ; fig. 4, PI. III). Le nombre de cellules FSH positives chez la Cane ovariectomisée (tabl. 1) en photopériode courte (5 405 cellules $/ \mathrm{mm}^{2}$ ) augmente de $50 \%$ par rapport au témoin. En photopériode longue $\left(6429\right.$ cellules $\left./ \mathrm{mm}^{2}\right)$, l'augmentation est de $100 \%$ par rapport à la Cane témoin et de $25 \%$ par rapport à l'animal ovariectomisé sacrifié en photopériode courte (fig. 4). Les paramètres taille et surface cellulaire projetés augmentent respectivement de $25 \%$ en photopériode courte et de $10 \%$ en photopériode longue par rapport à l'animal témoin (tabl. 1).

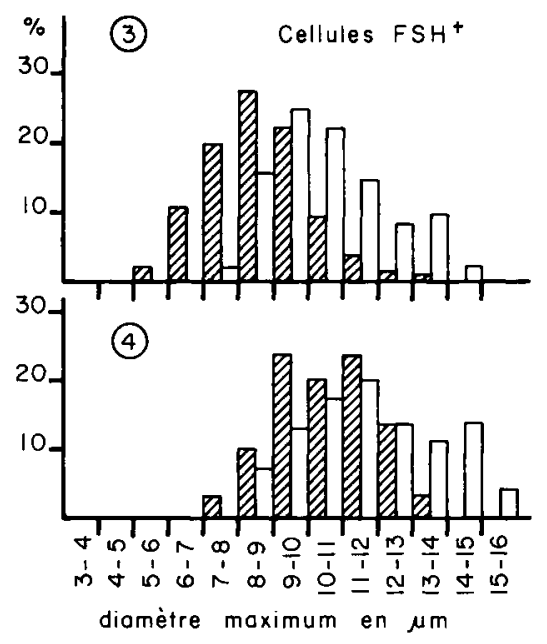

FIG. 3 et 4. - Histogramines de la distribution des cellules FSH ${ }^{+}$chez une Cane de Barbarie témoin (Fig. 3) et ovariectomisée (Fig. 4). Photopériode courte $Z Z Z$. Photopériode longue $\square$

2) Les cellules thyréotropes.

L'anti-bêta TSH bovine, du fait de sa spécificité immunologique, marque exclusivement les cellules thyréotropes qui se localisent dans le lobe céphalique de la pars distalis de l'hypophyse de la Cane de Barbarie. Chez la Cane témoin, en photopériode courte, le nombre de cellules TSH immunoréactives (64 cellules $/ \mathrm{mm}^{2}$ ) est inférieur à celui observé durant la période longue (178 cellules $/ \mathrm{mm}^{2}$ ) (tabl. 1). Les paramètres taille et surface cellulaire projetées sont réduits durant cette photopériode $(20$ à $30 \%)$.

L'ovariectomie s'accompagne d'une augmentation du nombre de cellules TSH immunoréactives (tabl. 1) chez les Canes sacrifiées en photopériode longue ou courte. Après ovariectomie, il n'existe pas de différence significative (test T) entre les paramètres cellulaires mesurés aux deux photopériodes. 


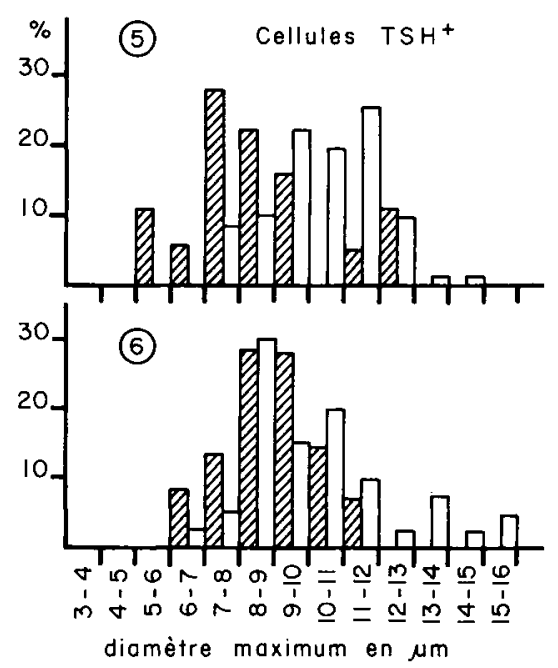

FIG. 5 et 6. - Histogrammes de la distribution des cellules $\mathrm{TSH}^{+}$chez une Cane de Barbarie témoin (Fig. 5) et ovariectomisée (Fig. 6). Photopériode courte $\square \backslash \lambda$. Photopériode longue

\section{Discussion - conclusion.}

Notre travail a permis de révéler, d'une part les cellules thyréotropes localisées exclusivement dans le lobe céphalique, et d'autre part les cellules gonadotropes LH/FSH qui se localisent dans les deux lobes de la glande pituitaire de la Cane de Barbarie. Ces cellules ont été mises en évidence en utilisant des anticorps dirigés contre les hormones ou parties d'hormones qu'elles élaborent. Seules les cellules contenant des grains de sécrétion sont immunoréactives; les cellules actives, mais vidées de leur contenu, ne sont pas visualisables. Rappelons que les hormones gonadotropes LH, FSH et thyréotropes des Vertébrés constituent une même famille de glycoprotéines (Fontaine, 1967). Les hormones gonadotropes ont été isolées et purifiées chez de nombreuses espèces d'Oiseaux, chez le Poulet (Stockell-Hartree et Cunningham, 1969 ; Scanes et Follett, 1972 ; Furuya et Ishii, 1974 ; Sakai et Ishii, 1980), chez la Dinde (Farmer et al., 1975 ; Burke et al., 1979), chez le Canard (Licht et al., 1977 ; Licht et Bona-Gallo, 1978), chez I'Autruche (Papkoff et al., 1982 ; Bona-Gallo et al., 1983 ; Licht $e t$ al., 1983).

Ces travaux montrent que ces glycoprotéines sont composées de deux sousunités distinctes : alpha et bêta. La sous-unité alpha est identique biochimiquement dans les trois hormones, mais l'activité hormonale spécifique dépend de la sous-unité bêta qui diffère dans les trois hormones. Par ailleurs, la parenté biochimique entre les gonadotrophines aviaires et les hormones de Mammifères semble bien établie (Papkoff et al., 1982 ; Bona-Gallo et al., 1983 ; Licht et al., 1983). Ceci étant, nous sommes en mesure de penser que l'anti-bêta TSH bovine que nous utilisons révèle de manière spécifique les cellules thyréotropes de l'adénohypophyse de la Cane de Barbarie. 
D'autre part, les anti-LH totale et anti-FSH totale de Poulet marquent principalement les cellules gonadotropes. Par ailleurs, chez les Oiseaux et chez la Cane en particulier (Sharp, 1974; Marchand et Bugnon, 1972 ; revue dans Marchand, 1978 ; Sharp et al., 1979 ; Mikami, 1980), les cellules thyréotropes sont toujours et exclusivement localisées dans la partie rostrale de la glande pituitaire. Donc, lorsque nous révélons des cellules immunoréactives vis-à-vis de l'anti-LH et/ou de l'anti-FSH dans la zone caudale de la glande totalement dépourvue de cellules thyréotropes, nous pouvons affirmer que ces cellules ne sont pas des thyréotropes, mais bien des cellules gonadotropes $\mathrm{LH}$ et/ou FSH. Cet avantage d'une localisation topographique préférentielle compense le défaut des anticorps totaux qui peuvent marquer à la fois les cellules gonadotropes et les cellules thyréotropes.

Nous pouvons conclure que les cellules thyréotropes du lobe céphalique et les cellules gonadotropes du lobe caudal forment deux populations cellulaires distinctes et numériquement bien séparées. Notre analyse révèle bien les fluctuations cellulaires en fonction des différents états physiologiques. Dans ces conditions, nous pouvons analyser l'effet de la photopériode et l'effet de l'ovariectomie.

a) La photopériode longue (avril-septembre) s'accompagne, chez les Canes témoins, d'une augmentation importante du nombre de cellules LH immunoréactives et de cellules TSH immunoréactives; le nombre de cellules FSH immunoréactives est inchangé. La taille et la surface cellulaire projetées des cellules TSH et FSH immunoréactives augmentent au cours de cette période lumineuse. Chez les animaux ovariectomisés, nous constatons l'augmentation importante du nombre de cellules gonadotropes et thyréotropes ainsi que des paramètres cellulaires mesurés, exception faite des cellules TSH positives.

b) A l'instar de ce qui est observé après castration chez le Canard mâle (Marchand, 1978), I'ovariectomie entraîne l'hyperplasie de l'adénohypophyse de la Cane de Barbarie, l'hypertrophie des cellules gonadotropes et des cellules thyréotropes.

L'analyse quantitative a le mérite de souligner la réponse importante de la population cellulaire gonadotrope aux stimuli lumière et ovariectomie, ainsi que I'hyperactivation des cellules thyréotropes et l'augmentation de leur nombre sous l'action de la lumière et/ou après ovariectomie. Nos observations permettent de penser à l'action freinatrice possible des stéroïdes ovariens sur la thyroïde, de façon indirecte, en contrôlant la libération de TSH par l'adénohypophyse, observations s'accordant avec les travaux d'Astier et al. (1970), Jallageas et Assenmacher (1979) effectués par dosages de T4 et de stéroïdes.

Reçu en août 1983.

Accepté en mars 1984.

\section{Références}

ASTIER H., HALBERG F., ASSENMACHER I., 1970. Rythmes circanniens de l'activité thyroïdienne chez le Canard Pékin. J. Physiol. Paris, 63, 219-230. 
BENOIT J., 1934. Activation sexuelle obtenue chez le Canard par l'éclairement artificiel pendant la période de repos génital. C. R. Acad. Sci. Paris, 195, 1671-1674.

BONA-GALLO A., LICHT P., PAPKOFF H., 1983. Biological and binding activities of pituitary hormones from the ostrich, Struthio camalus. Gen. comp. Endocr., 51, 50-60.

BURKE W. H., LICHT P., PAPKOFF H., BONA-GALLO A., 1979. Isolation and characterization of luteinizing hormone and follicle-stimulating hormone from pituitary glands of the turkey (Meleagris gallopavo). Gen. comp. Endocr., 37, 508-520.

DERAY A., 1978. L'ovaire dysgénésique de Cane hybride issue du croisement Pékin mâle (Anas platyrhynchos) par Barbarie femelle (Cairina moschata). Etude structurale et fonctionnelle des stades embryonnaires, aduites et sénescents. Comparaison avec les femelles des espèces parentes. Th. Doct. Etat, Besançon, $n^{\circ} 123,142 \mathrm{p}$.

FARMER S. W., PAPKOFF H., LICHT P., 1975. Purification of turkey gonadotropins. Biol. Reprod., 12, 415-422.

FONTAINE Y. A., 1967. Spécificité des glycoprotéines hypophysaires à activité thyréotrope et hypothèses sur leur phylogénie. Bull. Soc. Chim., biol., 49, 647-655.

FURUYA T., ISHII S., 1974. Separation of chicken adenohypophysial gonadotropins. Endocr. Japan, 21, 339-344.

GOMOT L., ARDIET P., 1966a. Inversion sexuelle progressive des gonades chez les femelles hybrides issues du croisement Canard Pékin $\times$ Cane de Barbarie. Bull. Soc. Zool. Fr., 91, 335-336.

GOMOT L., ARDIET P., 1966b. L'hybridation Canard Pékin $\times$ Cane de Barbarie. Etude comparée des Canards femelles Pékin, Barbarie et hybride. Ann. Sci. Univ. Besançon, 2, 125.

GOMOT L., ARDIET P., LUTZ-OSTERTAG Y., 1966. Intersexualité gonadique des hybrides femelles provenant du croisement Pékin mâle $\times$ Barbarie femelle. C. R. Soc. Biol., 160, $1572-1573$.

HANSEN G. N., HANSEN B. L., 1977. Light and electron microscopic identification of pituitary cells containing growth hormone and prolactin in the pigeon (Columba livia) using the immunoglobulin-enzyme bridge technique. Gen comp. Endocr., 32, 99-107.

HERLANT M., 1960. Etude critique de deux techniques nouvelles destinées à mettre en évidence les différentes catégories cellulaires présentes dans la glande pituitaire. Bull. Micr. appl., 10, 37-44.

HERLANT M., BENOIT J., TIXIER-VIDAL A., ASSENMACHER I., 1960. Modifications hypophysaires au cours du cycle annuel chez le Canard Pékin mâle. C. R. Acad. Sci. Paris, Ser. $D, 250,2936-2938$.

JALLAGEAS M., ASSENMACHER I., 1979. Further evidence for reciprocal interactions between the annual sexual and thyroid cycles in male Peking ducks. Gen. comp. Endocr., 37, 44-52.

LICHT P., PAPKOFF H., FARMER S. W., MULLER C. H., TSUI H. W., CREWS D., 1977. Evolution of gonadotropin structure and function. Rec. Prog. Horm. Res., 33, 169-248.

LICHT P., BONA-GALLO A., 1978. Immunochemical relatedness among pituitary follicle stimulating hormones of tetrapod vertebrates. Gen. comp. Endocr., 36, 575-584.

LICHT P., PAPKOFF H., BONA-GALLO A., AGGARWAL B. B., 1983. Subunits of an avian (ostrich) follicle-stimulating hormone and their hybridization with subunits of mammalian gonadotropins. Gen. comp. Endocr., 51, 414-422.

MARCHAND C. R., 1972. Recherches histophysiologiques sur l'adénohypophyse et les testicules des Canards hybrides stériles issus du croisement mâle Pékin $\times$ femelle Barbarie. Th. Doct. $3^{\mathrm{e}}$ cycle, Besançon, $\mathrm{n}^{\circ} 147$.

MARCHAND C. R., 1978. Les testicules et l'adénohypophyse des Canards de Barbarie /Cairina moschata L.) et hybride du croisement Canard Pékin $\times$ Cane de Barbarie. Etude structurale, histochimique, immunologique et expérimentale. Th. Doct. Etat, Besançon, $\mathrm{n}^{\circ} 126$.

MARCHAND C. R., BUGNON C., 1972. Caractérisation et localisation des cellules thyréoprives de l'adénohypophyse des Canards mâles et femelles Pékin, Barbarie et hybrides issus du croisement mâle Pékin $\times$ femelle de Barbarie. C. R. Acad. Sci. Paris, Sér. D, 274, 2335-2337.

MARCHAND C. R., BUGNON C., GOMOT L., 1969. Réponses cytophysiologiques de l'adénohypophyse à la castration des Canards mâles Pékin ou Barbarie et hybrides de ces deux genres. Ann. Sci. Univ. Besançon, 6, 30-35. 
MARCHAND C. R., BUGNON C., HERLANT M., 1975. Les cellules gonadotropes et les cellules à prolactine de l'adénohypophyse du Canard de Barbarie (Cairina moschata L. ). Etude immunologique. C. R. Soc. Biol., 169, 313-317.

MARCHAND C. R., BUGNON C., HERLANT M., DESSY C., 1976. Immunological characterization of the gonadotropic, lactotropic and corticotropic cells of the adenohypophysis of the Barbary duck (Cairina moschata). Gen. comp. Endocr., 29, n ${ }^{\circ} 68$.

MARCHAND C. R., BUGNON C., SHARP P. J., FELLMANN D., BLOCH B., 1978. Caractérisation immunologique et ultrastructurale des cellules gonadotropes, corticotropes, lactotropes et somatotropes dans l'adénohypophyse du Canard de Barbarie (Cairina moschata L.). Bull. Ass. Anat, 61e congrès.

MARCHAND C. R., SHARP P. J., 1977. Immunofluorescent localization and ultrastructural characterization of gonadotrophe cells in the adenohypophysis of the Barbary drake (Cairina moschata) using anti-chicken LH serum. Cell Tiss. Res., 181, 531-544.

MIKAMI S. I., 1980. The structure and function of the avian hypothalamo hypophysial system. Iwate Univ. Morioka, Japan.

MIKAMI S. I., VITUMS A., FARNER D. S., 1969. Electron microscopic studies on the adenohypophysis of the white-crowned sparrow, Zonotrochia leucophrys Gambelli. Z. Zellforsch., 97, 1-29.

MIKAMI S. I., KUROSU T., FARNER D. S., 1975. Light and electron microscopic studies on the secretory cytology of the adenohypophysis of the japanese quail, Coturnix coturnix japonica. Cell Tiss. Res., 159, 147-165.

PAPKOFF H., LICHT P., BONA-GALLO A., MCKENZIE D. S., DELFOSEN W., OOSTHUIZEN M. J., 1982. Biochimical and immunological characterization of pituitary hormones from the ostrich (Struthio cama/us). Gen. comp. Endocr., 48, 181-195.

SAKAI H., ISHII S., 1980. Isolation and characterization of chicken follicle-stimulating hormone. Gen. comp. Endocr., 42, 1-8.

SCANES C. G., FOLLETT B. K., 1972. Fractionation and assay of chicken pituitary hormones. Brit. Poultry Sci., 13, 603-610.

SCHWARTZ D., 1963. Méthodes statistiques à l'usage des médecins et des biologistes. Flammarion, Paris.

SHARP P. J., 1974. Immunofluorescent localization of pituitary cells in poultry using antichicken LH sera. Gen. comp. Endocr., 22, 365-366.

SHARP P. J., CHIASSON R. B., EL TOUSNY M. M., KLANDORF H., RADKE W. S., 1979. Localization of cells producing thyroid stimulating hormone in the pituitary gland of the domestic drake. Cell. Tiss. Res., 180, 53-63.

STERNBERGER L. A., HARDY P. H., CUCULIS J. J., MEYER H. G., 1970 . The unlabeled antibody enzyme method of immunohistochemistry. J. Histochem. Cytochem., 18, 315-333.

STOCKELL-HARTREE A., CUNNINGHAM F. J., 1969. Purification of chicken pituitary folliclestimulating hormone and luteinizing hormone. J. Endocr., 43, 609-616.

TIXIER-VIDAL A., 1972. Ultrastructural features of gonadotropic pituitary cells in culture. Symp. Biol. Hung., 14, 43-58.

TIXIER-VIDAL A., ASSENMACHER I., 1961a. Etude comparée de l'activité thyroïdienne chez le Canard mâle normal, castré ou maintenu à l'obscurité permanente. I. Période de l'activité sexuelle saisonnière. C. R. Soc. Biol., 155, 215-220.

TIXIER-VIDAL A., ASSENMACHER I., 1961b. Etude comparée de l'activité thyroïdienne chez le Canard mâle normal, castré ou maintenu à l'obscurité permanente. II. Période du repos sexuel saisonnier. C. R. Soc. Biol., 155, 286-290.

TIXIER-VIDAL A., BENOIT J., 1962. Influence de la castration sur la cytologie préhypophysáre du Canard mâle. Arch. Anat. micr. Morph. exp., 51, 265-286.

TIXIER-VIDAL A., GOURDJI D., 1965. Evolution cytologique, ultrastructurale de l'hypophyse du Canard en culture organotypique. Elaboration autonome de prolactine par les explants. $C$. $R$. Acad. Sci. Paris, 261, 805-808.

TIXIER-VIDAL A., FOLLETT B. K., 1973. The adenohypophysis, 3, 110-182. In FARNER D. S., KING J. R., Avian biology, Acad. Press, New York.

TIXIER-VIDAL A., HERLANT M., BENOIT J., 1962. La préhypophyse du Canard Pékin mâle au cours du cycle annuel. Arch. Biol., 73, 319-367. 
TIXIER-VIDAL A., FOLLETT B. K., FARNER D. S., 1968. The anterior pituitary of the japanese quail Coturnix coturnix japonica. The cytological effects of photoperiodic stimulation. $Z$. Zellforsch., 92, 610-635.

TIXIER-VIDAL A., CHANDOLA A., FRANOUELIN F., 1972. "Cellules de thyroïdectomie " et " cellules de castration " chez la Caille japonaise, Coturnix coturnix japonica. Etude ultrastructurale et cytoenzymologique. Z. Zellforsch., 125, 506-531. 